Homology, Homotopy and Applications, vol.10(3), 2008, pp.1-13

\title{
THE MATHEMATICAL WORK OF DOUGLAS C. RAVENEL
}

\author{
MICHAEL J. HOPKINS \\ (communicated by Donald M. Davis)
}

\begin{abstract}
This is a transcription of my talk on the work of Doug Ravenel. While it was intended as a one-time performance, the organizers felt it would be a good idea to have some representation appear as part of the conference proceedings. Don Davis went to the great trouble of transcribing the lecture from a video, and he sent it to me. After reading it I agreed to have it included in these proceedings. Even though the text is ungrammatical, repetitious and meandering (apparently that's how I talk), it feels to me as if it conveys something about how the world of algebraic topology has felt all these years with Doug in it. So with heartfelt thanks to Don Davis, here it is.
\end{abstract}

\section{The BP Mafia}

Gosh, when I came in Doug was just starting his talk, and he had all these slides and had the boards going, and I thought, "oh crap, he's put a lot more work into his talk than I have into mine." Then I started going through the notes for my talk and reading all the Math Reviews of his papers, and I thought, "oh crap, he's put a lot more work into my talk than I have."

When you go through the Math Reviews, the first paper you find of Doug's is something called $A$ definition of exotic characteristic classes of spherical fibrations, from the early 70s. No one ever reads that paper, and it's kind of forgotten. Doug wrote that when he was just a punk kid on the street. It was before he grew up and joined the BP Mafia. For those of you who are young enough to think that a Prius is a classic car, the BP Mafia is rumored to consist of Haynes Miller, Doug "Whitey" Ravenel, and Mr. Wilson. It's not really fair to call this a "mafia." You see, a "mafia" refers to an Italian group in organized crime, and these guys were strongly influenced by a certain Greek element.

These guys are famous for the amazing work they did in an amazingly short period of time using Brown-Peterson homology to do important calculations in homotopy theory. We've heard many examples of talks today about the influence of those ideas and what those things have grown into. I wanted to try to say a little bit about what this body of work is.

Received April 3, 2008, revised September 7, 2008; published on December 5, 2008. 2000 Mathematics Subject Classification: 55T15, 55N22, 55Q51.

Key words and phrases: Adams-Novikov spectral sequence, stable homotopy theory, jokes.

This article is available at http://intlpress.com/HHA/v10/n3/a1

Copyright (c) 2008, International Press. Permission to copy for private use granted. 


\section{Adams periodicity and self-maps}

The story probably starts with the introduction of the Adams spectral sequence. Adams introduced this spectral sequence that goes from the cohomology of the Steenrod algebra and converges to the homotopy groups of spheres. The first thing you have to prove about it is that it really converges. To see what Adams really did, I'll draw the Adams chart in the usual way.

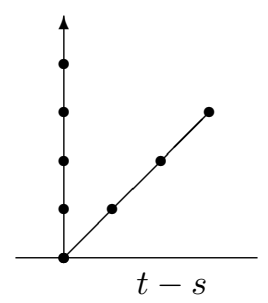

The first thing that he proves is that if you get rid of this tower, then everything above a line of slope 1 is 0 . That's called the Adams vanishing line, and it was the key to showing the Adams spectral sequence converges.

When you move this line of the same slope down a little bit, you see a little pattern. You see this little recurrent pattern of groups over and over - a little pattern of three homotopy groups that repeats itself every 8 dimensions.

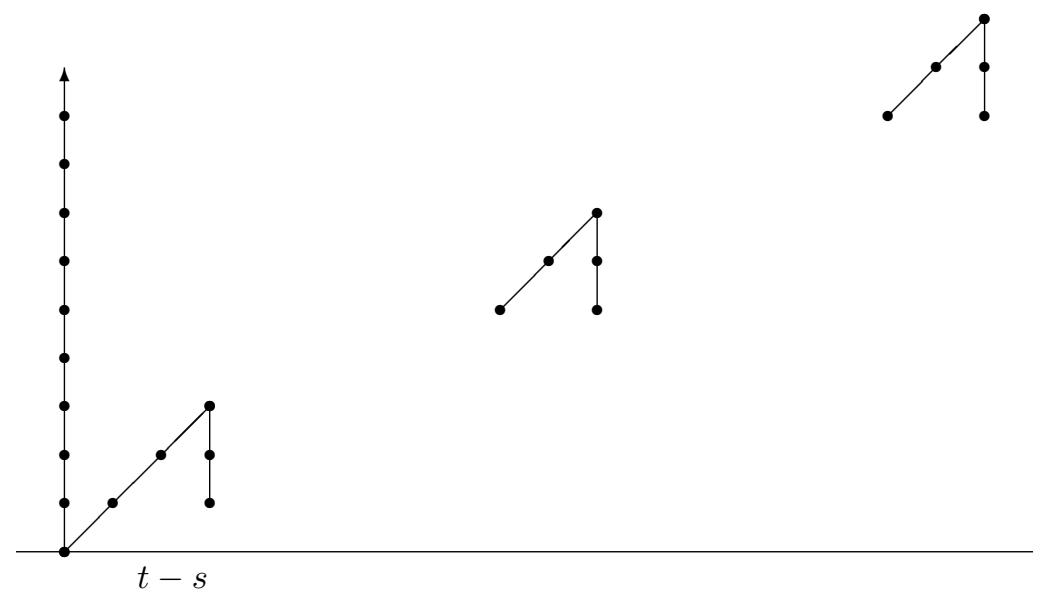

The fact of its repeating was a rather striking feature of the cohomology of the Steenrod algebra. This was something that was called Adams periodicity. It reflected some kind of strange recurrent phenomena in the homotopy groups of spheres. Adams did quite a lot with this. He recognized it was connected to Bott periodicity. It reappears in his analysis of the image of the $J$-homomorphism, and he later explained it in his $J(X)$-papers by producing a self-map from the 8-fold suspension of the mod-2 Moore space to itself that's an isomorphism in $K$-theory

$$
\Sigma^{8} S^{0} \cup_{2} e^{1} \rightarrow S^{0} \cup_{2} e^{1},
$$

and also an analogous self-map from the $(2 p-2)$-fold suspension of a $\bmod p$ Moore 
space

$$
\Sigma^{2 p-2} S^{0} \cup_{p} e^{1} \rightarrow S^{0} \cup_{p} e^{1}
$$

So what does that have to do with these elements? Well, he also introduced this great trick. This map can be iterated as many times as you want, and it will never be null because it is an isomorphism in $K$-theory. I like to draw it like this, because I learned this stuff from Mahowald.

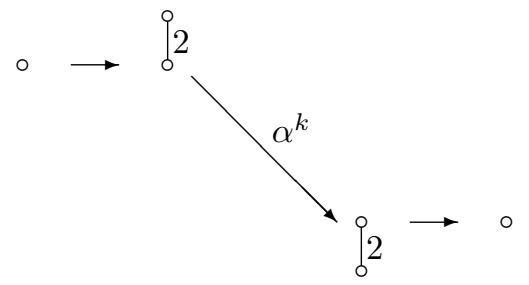

You represent your 2-cell complex by a picture of two circles with the attaching map indicated. You iterate Adams' map. You restrict to the bottom cell of the top one and pinch to the top cell of the bottom one. Let's call the original map $\alpha$, and its iterates $\alpha^{k}$. As $k$ varies, you get a sequence of elements in the homotopy groups of spheres, and these correspond to moving along the Adams edge in our chart. They are a geometric manifestation of Adams periodicity. Adams had a lot to do with our understanding of the homotopy groups of spheres, and was the first to really see this mysterious recurrent pattern.

\section{Greek elements}

I guess I don't quite know the timing of all this, but around the late 60s, Larry Smith and Hirosi Toda sought to generalize this. So they replaced $K$-theory with $B P$. They wanted to generalize Adams' complex, so they looked for certain complexes $V(n)$ for which

$$
B P_{*}(V(n)) \approx B P_{*} /\left(p, v_{1}, \ldots, v_{n}\right) .
$$

They looked for a self-map

$$
\Sigma^{2 p^{n+1}-2} V(n) \rightarrow V(n)
$$

that induces multiplication by $v_{n+1}$ in $B P$-homology. This is not a divisor of 0 in $B P$-homology, so this map won't be nilpotent, and you can get a similar family of elements in the homotopy groups of spheres.

When $n=0$, this family is the family that Adams discovered, and in honor of Adams, we call these $\alpha_{1}, \alpha_{2}$, etc. When $n=1$, we needed a new letter, and after $\alpha$ comes $\beta$. So we called these $\beta_{1}, \beta_{2}$, etc., and on through the Greek alphabet. So $\gamma_{1}$, $\gamma_{2}$ were the next ones, and this is about as far as people could get.

$$
\begin{array}{ll}
n=0 & \alpha_{1}, \alpha_{2}, \ldots \\
n=1 & \beta_{1}, \beta_{2}, \ldots \\
n=2 & \gamma_{1}, \gamma_{2}, \ldots
\end{array}
$$

No one could really construct very many of these complexes. In fact, I think we 
believe that not very many of them exist. Anyway, this was already enough infinite families of elements in the homotopy groups of spheres to try to come to grips with. In anticipation of the program going on, these were called the Greek letter elements. And just so they wouldn't get to $\Omega$ and wonder what the heck to do, folks decided to call the $n$th Greek letter " $\mathrm{gr}_{n}$." It's not obvious that the elements you get this way are nonzero. One question that Smith and Toda were worried about was whether the $\gamma$ 's were nontrivial.

\section{The work of the BP Mafia}

That's the problem that the BP Mafia took up and analyzed, using ideas of Jack Morava, in this fantastically beautiful way. They recognized that we need to study the Adams-Novikov spectral sequence, i.e., that we need to study

$$
\operatorname{Ext}_{B P_{*} B P}\left(B P_{*}, B P_{*}\right) \Rightarrow \pi_{*}\left(S^{0}\right) \text {. }
$$

Already just recognizing that this is the object to study is a little bit nontrivial. One of the things that at the time was a little bit novel was that they were working with homology instead of cohomology. All of the psychology of the classical Adams spectral sequence had been in terms of the cohomology of the Steenrod algebra. Here these guys changed emphasis and worked with homology and gave more manageable formulas. Raph Zahler had been trying to understand the spectral sequence from the point of view of cohomology. The shift to homology, which we take for granted now, was already a good idea. But what was really amazing was the way they organized this calculation. Let me try and portray that a little bit, and then start listing some of the papers.

They used the theory of formal groups, and, inspired by ideas of Morava and Quillen, came up with the following maneuver, the following way of getting at these Ext groups. You take $B P_{*}$ and invert the first guy,

$$
B P_{*} \rightarrow p^{-1} B P_{*},
$$

and take the cokernel. If you just look at multiplication by $p^{n}$, that would be $B P_{*} / p^{n}$. I am doing this infinitely many times. Topologists like to call this $B P_{*} / p^{\infty}$. Then you invert $v_{1}$. Take the cokernel of that map. Then you invert $v_{2}$, etc.

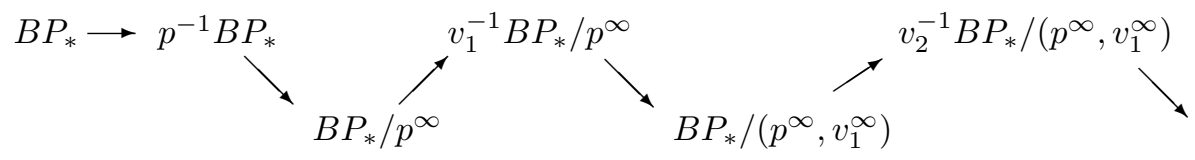

Filling in the horizontal arrows, you get this complex,

$$
B P_{*} \rightarrow p^{-1} B P_{*} \rightarrow v_{1}^{-1} B P_{*} / p^{\infty} \rightarrow v_{2}^{-1} B P_{*} /\left(p^{\infty}, v_{1}^{\infty}\right) \rightarrow,
$$

this resolution for computing Ext for $B P_{*}$. This leads to a spectral sequence that goes from

$$
\bigoplus \operatorname{Ext}_{B P_{*} B P}\left(B P_{*}, v_{n}^{-1} B P_{*} /\left(p^{\infty}, v_{1}^{\infty}, \ldots, v_{n-1}^{\infty}\right)\right)
$$

and converges to the thing, $\operatorname{Ext}_{B P_{*} B P}\left(B P_{*}, B P_{*}\right)$, that you were interested in. 
This was a very interesting object already, but, inspired by the work of Morava, these guys simplified this with two maneuvers. This $v_{n}^{-1} B P_{*} /\left(p^{\infty}, \ldots, v_{n-1}^{\infty}\right)$ is like $\mathbb{Q} / \mathbb{Z}$, which is a direct limit of $\mathbb{Z} / n$. It's a big direct limit where you have modded out finitely many powers of each of these, and each of those can be filtered so that you have just modded out a single one. You can understand this by some elaboration of the Bockstein spectral sequence if you can understand

$$
\operatorname{Ext}_{B P_{*} B P}\left(B P_{*}, v_{n}^{-1} B P_{*} /\left(p, \ldots, v_{n-1}\right)\right) .
$$

This is all good. Then they add to the mix one more brilliant isomorphism, which is that this is isomorphic to

$$
\operatorname{Ext}_{K(n)_{*} K(n)}\left(K(n)_{*}, K(n)_{*}\right),
$$

and this is something you can compute.

Just looking at $\operatorname{Ext}^{0}$ here - those are the elements in this ring that are invariant under all this, so elements in $\operatorname{Ext}^{0}\left(v_{n}^{-1} B P_{*} /\left(p^{\infty}, \ldots, v_{n-1}^{\infty}\right)\right)$ (here we abbreviate with the only symbol that's varying in this expression) contribute to

$$
\operatorname{Ext}_{B P_{*} B P}^{n}\left(B P_{*}, B P_{*}\right),
$$

and the image of these Ext $^{0}$ elements are these Greek letter elements. We should probably call it the "Greek letter family," in honor of the Mafia. So that's how these guys organized the Greek letter family, and ultimately made many many important calculations.

\section{Their papers}

I want to give you an idea of the amazing amount of progress that was made in an amazingly short period of time. I was going to write the names of all these papers, but I'm worried that that's going to go a little slow. I'm going to list the date the papers appeared, but I know from personal experience that that has nothing to do with when the research was done.

1975 MRW Novikov's Ext $^{2}$ modulo an invariant prime ideal and nontriv-
iality of the $\gamma$ family

This was the first paper when Miller, Ravenel, and Wilson were introducing these ideas - the first announcement. There is actually a famous controversy surrounding this, but I think Martin is going to talk about that - some of the controversial antics of the BP Mafia.

In the same year, there is another paper of Doug's

$1975 \mathrm{R} \quad$ Multiplicative operations in $B P^{*} B P$

and in 1976, there is another paper of Ravenel

$1976 \mathrm{R} \quad B P_{*} B P$ modulo an invariant prime ideal

Then there's these two fantastic companion papers, again by Ravenel,

1976 R Structure of Morava stabilizer algebras

1977 R Cohomology of Morava stabilizer algebras 
Then in 1977

1977 MR Morava stabilizer algebras and localization of Novikov's $E_{2}$

now called the Change of Rings paper. This was all happening in one to two years, and then came the raison d'etre of all these papers, the famous Annals paper which appeared in 1977:

1977 MRW Periodic phenomena in the Adams-Novikov spectral sequence

I put all these up partly to make you feel bad. If you've ever had a day at the end of the year when you have to write about your progress that year on your NSF grant, and you're just hoping someone wrote a paper and put your name on it, because, God knows, you can't think of any. Look at this. In this two-year period, Doug has written all these papers, and that's only on this topic. There's another topic I'm going to get to in a minute. He's got 12 papers that came out-Boom!-all at once, and they're all fantastic. It makes me feel terrible, but I've gotten used to the idea because I've been thinking about this talk for a couple of days.

One of the things that I find is a great lesson: We know what these ideas grew into, and I'll be talking about that more, but they grew into all this stuff you've heard about today. All these structures are still with us, in fancier and fancier forms. We've heard about different Ext groups. We've heard about topological modular forms and topological automorphic forms, and all these things came out of people riffing on this circle of ideas. What was it that these guys thought was the important thing that they did? They thought the important thing they did was establishing the nontriviality of the $\gamma$-family. To us, that doesn't seem nearly like the most interesting part of it. I'm harping on this because it is a real lesson about doing mathematics, and about the evolution of mathematics. This is three brilliant mathematicians narrowly focused on a good question, and they answered it in this amazing way. When you are trying to come up with problems for yourself, for your own research, just remember that: break off concrete and real questions. Take this as a guide. Look at all that amazing stuff that came out of this circle of ideas.

\section{Math Reviews}

We think this stuff is amazing. Not everyone thought it was amazing. I want to read to you from some of the Math Reviews. This is what's great. You really suffered, Doug! Most of your papers in this period were reviewed by Adams. ${ }^{1}$ This is the review of the big one, the Periodic Phenomena in the Adams-Novikov Spectral Sequence. Adams goes on and explains the circle of ideas, but here's how "big" he thought this was. He concludes his review with "This paper contains a great deal of factual information based on detailed calculation of a sort difficult to summarize in a review. One can say though that $\operatorname{Ext}_{B P_{*} B P}$ " (and he mentions some numbers where they computed it) "and that there is some information, even if it is not complete, for $s=3$, and also for $p=2$; and that this gives commendable information on infinite families in stable

\footnotetext{
${ }^{1}$ After my talk Francis Clarke privately objected that I had been unfair to Adams, who had meant his comments to be tongue-in-cheek. I was, of course, aware of this, but succumbed to the temptation of taking them literally just to have more fun. That seemed OK at the time, but now that this is going into print, I figure I'd better come clean.
} 
homotopy." Here's the zinger: "This paper is clearly required reading for experts on odd-primary stable homotopy." I love that!

Here's Adams commenting on the Structure of Morava stabilizer algebras paper. He talks about all these great preprints of Morava that were floating around, and how these guys put parameters in, put coordinates in so you could really write down calculations, and he says "No doubt topologists will welcome a version which can be read by those who are not familiar with modern algebraic geometry." I like that because the way we eventually wanted to understand this was through the language of algebraic geometry.

I wanted to just point out a little bit about how this looked even in the day. That's supposed to be inspiration. If you feel like you are focused on a narrow question and the big shots aren't interested in it, and they have it relegated to those specializing in homotopy theory at regular primes, take hope!

These ideas continued to grow in many ways. The Miller-Ravenel Change-of-Rings theorem is the isomorphism involving (1). This we kept reunderstanding in different terms and eventually saw this in the spirit of Morava's vision using the language of stacks. It's an amazing testimony to the power of Doug and his collaborators' ability to understand these things. They were able to just write down elements and get this isomorphism.

If you study this stuff, and I imagine if you are here you have, you've looked at all these $B P$ formulas - the right unit, the coproduct, the log, the way you organize all these things. It's amazing. I once asked Doug "How long did it take you to come up with all those formulas? I never could have done it. I could barely understand the theory." And he said "Oh, a couple of weeks." It happened very very quickly. There's an amazing density of ideas here.

\section{Hopf rings}

There's a lot more to talk about. There's another idea that comes along about the same time which is another tool in studying these things, which Doug and Steve called Hopf rings. We heard about Hopf rings and their generalizations in Sarah's talk today. People are still thinking about these ideas. I'm worried I'm going to run out of time for what I want to say, so I'll go a little bit quickly through Hopf rings.

This started with Steve Wilson's thesis. But the general question is: Suppose I have a ring spectrum $E$, and $\underline{E}_{n}$ are the spaces in the loop spectrum, so that $\Omega \underline{E}_{n+1}=\underline{E}_{n}$. Then if I have some homology theory, like ordinary homology $\mathcal{H}$, the $\mathcal{H}_{*}\left(\underline{E}_{n}\right)$ have a highly elaborate algebraic structure. These form what Ravenel and Wilson called a Hopf ring. This is a remarkably tight amount of structure.

You have algebraic maps that reflect the product. These are coalgebras. You have something reflecting the sum operation and the product operation. And you have all these Rothenberg-Steenrod spectral sequences that relate the homologies of these to each other. This turned out to be such a surprisingly rigid amount of structure, they were able, as if by magic, to calculate many many Hopf rings. In particular, they calculated $\mathcal{H}_{*}(\underline{M U})$ and wrote down a universal formula for that in terms of formal groups. 
Doug and Steve have a few papers here. I'll just put down three of them to add to this list in this amazingly productive period.

1974 RW Bipolynomial Hopf algebras

1974 RW The Hopf ring for complex cobordism

1974 R Dieudonné modules for abelian Hopf algebras

You should have saved some of these for the lean years. I'll come back to some of these ideas a little bit later.

They calculated the Hopf ring for $M U$, and they do it for many other homology theories. In their hands, these ideas continued to grow, and a lot of us were inspired by this. John Hunton and I were inspired by this, and years later showed that for any Landweber exact homology theory the Hopf ring has an analogous description in terms of the formal group. Then Paul Goerss, using Doug's Dieudonné module paper, found an extremely beautiful proof of that. And you just heard Sarah Whitehouse talking today about these ideas. These were beautiful papers about concrete problems, but they inspired us for thirty years.

Again, I'm not the only one who has an opinion about these papers. Here's a good one. In The Hopf ring for complex cobordism, where these guys calculate all the homology of the spaces in the loop spectrum for $B P$, Adams concludes his review with "This paper seems to be required reading for $B P$-specialists." That's another good one.

\section{Odd-primary Arf invariant}

Here's this amazingly productive period. Twelve papers in a couple of years on a range of topics. They've settled the $\gamma$ family stuff, they've made all these important calculations in homotopy theory, and developed this amazing machinery. So then Doug moves on to apply these techniques to other problems. There is one in particular I want to mention. This is a 1978 paper called The nonexistence of odd-primary Arf invariant elements in stable homotopy. In this paper, Doug proves that there are no odd-primary analogues of Arf invariant 1. I found this paper extremely inspiring when I read it because he takes this huge complicated Novikov $E_{2}$-term Ext over $B P$, and he maps it to the cohomology of the cyclic group $\mathbb{Z} / p$ and detects some phenomenon that he wants to detect. It stayed in my head for a long time. When I read it, I was in graduate school, and I realized what it meant was that any time you have a cyclic group, a finite group acting on a formal group, you have a map from the Novikov $E_{2}$-term to the cohomology of that group. I mention this because I think in Doug's hands this was an application of this circle of ideas, a natural outgrowth of this. This paper was the one that led Haynes and me to consider what became known as Hopkins-Miller theory.

It was this argument that made us realize that the finite subgroups of Morava stabilizer groups are important, and that's what led to TMF and TAF and all these things. So again for me personally this paper of Doug's on the Arf invariant was a really fantastic paper. You can understand this one before you understand many of the earlier papers. This is a really good gateway into this stuff. The Math Review of this paper has a really good line in it. I don't know what people were smoking back then. Actually I do know what people were smoking back then. This isn't Adams. 
The review says "This paper concerns the Adams-Novikov spectral sequence thought of as localized at a single odd prime in terms of $B P \ldots$ The title, which is an informal rather than precise description of its contents, ..." Informal?

By the end of the 70s, the BP Mafia are established. They are pretty well-known and pretty well respected. Ravenel gives a talk at the Helsinki ICM. Another paper that appears at this time is called A novice's guide to the Adams-Novikov spectral sequence. Here's Adams' review, finally coming around. "This is a good, lively exposition of the applications of BP-theory to stable homotopy theory, which one associates with such names as H. R. Miller, D. C. Ravenel and W. S. Wilson." He's finally come around. And then just to show you that there is a time-honored tradition of calling things "classical" that really rankles, he goes "the author discusses the "classical' Adams spectral sequence," and then ends the review saying "Some students of nature like to watch exotic ducks, and some to observe homotopy elements known by the less familiar Greek letters." So that's the Greek letter story, and that's these guys.

\section{The Ravenel conjectures}

There is an embarrassment of riches in this work, and I am actually going to have to skip some stuff, because I can't squeeze a talk on the work of Doug Ravenel into an hour. I have to talk about the part that impacted me the most, which is a paper that Doug wrote that circulated in preprint form in the early $80 \mathrm{~s}$. I'm going to move on to that.

After these spectacular applications and this amazing computational apparatus arose, Doug turned to the question of trying to understand what these algebraic maneuvers had to do with homotopy theory. He didn't want them to be just algebraic. He wanted all this stuff, the families, the periodicity, to be manifest with some geometric aspect of stable homotopy. He wrote this paper in 1984 called Localization with respect to certain periodic homology theories. It circulated in preprint form, and in it he made a bunch of conjectures.

I got hold of this paper in a funny way. I was a graduate student in Oxford. This must have been about '82-83. I spent a lot of time talking to Zdzislaw Wojtkowiak, who was in exile from Poland. One day he came up to me. At the time he had a way of using English that made it seem like he had already hypnotized you. He said "Mike, you will send to Doug Ravenel a letter asking for a copy of his paper," and he named it. I'm starting to obey (walks as if hypnotized) and suddenly I stop myself and say "Wait a minute! Why do you want me to do that?" He says he had written for one and he got it but he lost it. I said "Why don't you just ask him for another one?" and he said "I can't." So then I knew that Doug Ravenel, who I never really heard of before, was a really scary monster guy. I wasn't going to write for a paper like that. But finally he twisted my arm. So I said "I'm going to do it just once, so what other papers does this guy have? I'm not going to write to him twice."

So I wrote to Doug and asked for that paper, and I figured I was a novice, so I also needed A novice's guide to the Adams spectral sequence. I got a few other papers. Zdzislaw and I talked a lot about them. The next year I was at Northwestern, and I still didn't understand things very well. I met Jeff Smith there, and Jeff explained the localization paper to me. I think I've never been more interested in a paper in my 
life. This paper-I'm sorry, I'm getting choked up - really really inspired me. Those days with Jeff, and when we met Ethan, it was really magical.

Anyway, at Northwestern Jeff and I were always talking about the Ravenel conjectures. I was still worried about this character Ravenel. He even had kind of a scary name. Roy Joshua was around, and it turned out Ravenel was going to come and give a talk. I cornered Roy and asked "Have you met Ravenel before?" and he says "Yeah," and I say "Is he really scary?" Now that's one of those questions that you can't really answer "No" to, just in case you're wrong. He said "Yeah, he's really scary." I don't remember, but I think I might have skipped his talk.

So that's how I got hold of Doug's paper, and how I learned about the Ravenel conjectures. I finally met Doug too, who turned out not to be so bad. Eventually Jeff and Ethan and I proved the conjectures in this paper. It was a very exciting time, and I am very grateful to Doug for writing that paper.

Maybe I should say a little about what's in it. In this paper, he tries to take what the BP Mafia did, and render it geometric. He makes a series of inspiring conjectures about homotopy theory. There is something I have to say about that era. This was in the early 80s. If you are coming into the subject and you are a graduate student, you love the machinery, you love the tools, but the people who came to it before you are people like Doug and Haynes and Steve and Mahowald and Adams and Peter May and Fred Cohen - all these people that did all these amazing calculations. We just knew that we were never going to be able to outcompute these guys, and we needed some different opportunity, some place else to sink our intellect into. And that's probably the reason this paper was so amazing. Because it was about qualitative global aspects of homotopy theory, and it wasn't hinging on the details of calculations. This was really just the place a young person could get going.

In this paper, he makes a number of conjectures about the stable category. But the idea, as I said, is to render in geometric terms the chromatic story. Among the conjectures in the paper - there are many - are:

One of them concerns the existence of complexes like this $V(n)$. These were extremely hard to come by. Finding one of these was like finding the triple platinum gem at level 3 of your favorite Sony Playstation game. Doug had the amazing idea that if you ask for a little less, these things were all over the place. What he actually conjectured was that if $X$ is a finite spectrum and $K(n-1)_{*}(X)=0$, then there is automatically a map $\Sigma^{N} X \rightarrow X$ which is an isomorphism in $K(n)_{*}(-)$. In particular, you could iterate this map and it was not nilpotent.

Another conjecture was that if a self-map of a finite spectrum is 0 in complex cobordism, it is nilpotent; i.e., complex cobordism detects nilpotent maps. He also brought many more tools into this. He brought into the game the theory of Bousfield localization. There was another thing in this paper called the Telescope Conjecture, which says that if we call the map $\Sigma^{N} X \rightarrow X \rho$, then

$$
\rho^{-1} X \simeq L_{K(n)} X \text {. }
$$

The spectrum on the left is obtained by iterating $\rho$ and taking the colimit, while the one on the right is Bousfield localization with respect to $K(n)$.

There are many more things. I don't have time to describe all the conjectures that were in the paper. They were very inspiring. There had never been a vision like that 
written about homotopy theory. Ethan, Jeff, and I proved these conjectures. The three of us proved the one about nilpotence. Doug was out in Seattle at the time. Jeff and I were on our way to spend a semester in Seattle, and drove from Chicago, and on the drive out, we proved the rest of them. It was a fantastic road trip. If I didn't have a bad back, I'd try to do it again. We were able to prove all of them except for the Telescope Conjecture, and it remains open to this day.

\section{A colloquium talk}

At the time, I was asked to give a lot of colloquium lectures on this stuff. It was always a little bit tough because I had to talk about Morava $K$-theory, I had to talk about spectra, I had to talk about Bousfield localization. I figured I could say $M U$, and not mention $B P$, but it was a lot of stuff. I would always begin my talk a little bit hopeful by saying "Are there any experts on odd-primary stable homotopy theory?" But I never had a Yes.

But there's a way I eventually found a couple of years ago that I really liked to describe it. You really can't explain it. You can convey the impact of this to a relatively general mathematical audience. In fact, I described it this way at a conference in honor of Chern, so there were a lot of geometers in the audience. I talked about Chern's calculation of the first Chern class of a line bundle, and how we look at cohomology theories for which that calculation holds, complex-oriented theories, and we look at what maps between things these theories try to detect. We filter our world of maps. You say a map $X \rightarrow Y$ has filtration $n$ if you can filter it through $n$ maps which are not seen by any of these Chern theories. To us that means it has Adams filtration $n$ in the Novikov spectral sequence. So you can make a chart of the homotopy groups of spheres, and look at the highest filtration in the $n$-stem. Call it $g(n)$, and you get a curve, which I like to call the vanishing curve.

All these conjectures are equivalent to

$$
\lim _{n \rightarrow \infty} \frac{g(n)}{n}=0 .
$$

It would be really interesting to know what the first term in the asymptotic expansion of $g$ is. We have no techniques for dealing with it. The question is: if $g(n) \sim c \cdot n^{\epsilon}$, what is $\epsilon$ ? If you think of $\epsilon$ as a parameter, you can dial it like Planck's constant.

It's kind of fun to think about what the world looks like with different values of $\epsilon$. If $\epsilon$ is really small, this line is really steep, and the Telescope Conjecture is automatically true. If $\epsilon$ is big, we don't get as much information. Jeff Smith and I came up with a plausibility argument that $\epsilon$ should be $\frac{1}{2}$. There is actually an easy way to convey that, because Lie groups in their left invariant framing give you stable maps between spheres. The filtration for a Lie group is at least its rank, and the rank is about the square root of dimension for all the infinite families, so that makes you think that $g(n)$ is something like $n^{1 / 2}$.

That's kind of a hoax. We think the Lie groups are mostly 0 in homotopy theory. But nevertheless I just want to advertise this. After all these years thinking about this, this is somehow the next step - to figure out what is $\epsilon$, and what does it really mean for homotopy theory. If $\epsilon=\frac{1}{2}$, it doesn't tell you anything about the Telescope Conjecture. Nobody said we were happy people. 


\section{Character theory, and more}

I am embarrassed by the riches. There is so much stuff of Doug's that I didn't talk about. I didn't talk about our character theory. One of the things I didn't talk about, that Nora mentioned in her talk, was the generalized group characters that Doug, Nick, and I came up with. That's a better example of realistic publication time. Originally Doug gave a talk in 1981 at the Adem conference proving that the dimension of the Morava $K$-theory of $B G$ is finite. A couple of years later in New Orleans, he gives a lecture guessing that its dimension is about equal to the number of $n$-tuples of commuting elements. After Nick, Doug, and I proved all this, I asked Doug where the paper was. How come he gave that talk and there was no paper? He said, well, he thought it might be true, and he wanted to make himself prove the theorem. So he announced the title of the talk a couple of months before the talk, figuring that the pressure of that would make him prove the theorem, and it really worked. So I just wanted you to know if I hadn't been forced to give this talk, I was going to announce a proof of the Riemann hypothesis.

We announced the stuff about the character theory at Ed Brown's 60th birthday in 1986, and then a paper appears in 1992 that's kind of another announcement of the theorems, and the final paper finally appeared in JAMS in 2000.

There's so much more - so many amazing calculations. Doug has been extremely generous with young people, and even not-so-young people. One time in the mid 90s, I was talking to Neil Strickland about Morava $K$-theory of things. He was dealing with finite Postnikov towers, and he wrote down this amazing formula. I said "That's cool. Where did you learn that?" and he goes "In that paper of yours." I go "What paper of mine?" and I look and there's this paper of Hopkins, Ravenel, and Wilson on Morava $K$-theory of finite Postnikov towers. I'm really glad that I'm an author of that, but I honestly don't remember. Anyway, that was very nice.

Doug had work on the Segal Conjecture, and he still continues to do amazing calculations, some of which you just heard.

Here is a recent Math Review of a paper of Doug's called On the generalized Novikov first Ext group modulo a prime. The reviewer here is none other than Steve Wilson, and he writes of his dear friend "The main result of this paper is to compute ... Results are a bit technical, but it is fascinating to compare similarities and differences between ..." So even these guys are slowing down.

\section{A big giant round orange head}

There is a great joke I heard: A guy is walking down the street, and he sees a friend of his with a big giant round orange head. He says "What happened to you. How did you get a big giant round orange head?" The guy says "I was walking in the desert, and I found this lamp. I was dusting it off to see what it was, and a genie came out and offered me three wishes. First I asked to be wealthy. I got a huge amount of money. There's my mansion over there. For my second wish, I asked for a really beautiful wife, and there she is. Look at all those people hanging around her. For my third wish, which is where I think I went wrong, I wished for a big giant round orange head." 
Now - algebraic topology — how we got here. First people studied homotopy groups of spheres with geometric methods. All you can learn about homotopy groups of spheres with geometric methods is that the homotopy groups of spheres are countable. If you want to know that they are finitely generated or finite, you have to introduce Eilenberg-MacLane spaces. What you need to know is that you got rid of all the homotopy groups by putting in finitely many cells in every dimension.

Eilenberg-MacLane spaces already cut us off from the geometric world. They are what I now think of as designer homotopy types. If you're in the subject, you love them; if you're outside, they are kind of mysterious. Doug and his friends gave us a fantastic collection of these designer homotopy types.

$$
B P, T(n),
$$

which Doug used to calculate $\pi_{*}(S)_{(5)}$ out to the 1000-stem,

$P(n), E_{n}, K(n)$, Hopf rings, Brown-Comenetz duals, Bousfield localizations,

all these designer homotopy types. We owe all these guys big thanks. But I want to personally tell Doug thanks for all the beautiful ideas and all the rich things to think about and thanks for the big giant round orange head.

Michael J. Hopkins mjh@math.harvard.edu

Department of Mathematics, Harvard University, Cambridge, MA 02138, USA 